\title{
Update on cesarean on request
}

\section{AtualizaçÃo EM CESARIANA A PEDIDO}

Sergio F. de Toledo ${ }^{1}$, Ricardo Simões ${ }^{1,2}$, Romulo P. Soares ${ }^{2}$, Luca S. Bernardo ${ }^{2}$, Wanderley M. Bernardo ${ }^{2}$,

Antônio J. Salomão ${ }^{1}$, Edmund C. Baracat ${ }^{1}$

1. What is the correct approach to maternal request for cesarean section?

a. Know with greater emphasis personal values and preferences of the mother and approach them in a process of shared decision.

b. Perform cesarean delivery without questions and explanations, since this is the patient's will.

c. Disregard the patient's request, since this is a decision of the doctor exclusively.

d. Show scientific evidence of the benefits of normal birth without sharing decision with the patient.

2. Cesarean section on maternal request or with no indication increases the risk of bleeding complications?

a. Yes, it increases the absolute risk of bleeding complications with statistical significance.

b. Yes, it increases the risk of complications, but without statistical significance.

c. No, there were no differences in the evaluated results.

d. No, vaginal delivery increases the absolute risk of bleeding complications in a statistically significant manner.

3. Cesarean section on maternal request or with no indication increases the risk of infectious complications?

a. Yes, it increases the absolute risk of infectious complications with statistical significance.

b. Yes, it increases the risk, but without statistical significance.

c. No, there were no differences in the evaluated results.

d. No, vaginal delivery increases the absolute risk of infectious complications in a statistically significant manner.

4. Cesarean section on maternal request or with no indication increases the risk of which outcomes?

a. Hemorrhagic, infectious and breastfeeding complications, and respiratory complications for the newborn.

b. Hemorrhagic and infectious complications, only.

c. Maternal mortality, surgical wound complications, neonatal asphyxia, neonatal infection and hospitalization in the neonatal ICU.

d. Bleeding complications, maternal mortality, breastfeeding complications, and neonatal infection.
5. What is the period when the cesarean section should be done in case of vaginal delivery refusal?

a. After 39 weeks.

b. After 37 weeks.

c. After 42 weeks.

d. Before 38 weeks.

\section{ANSWERS TO CLINICAL SCENARIO: BIRTH ROUTE IN CASE OF CESAREAN SECTION IN A PREVIOUS PREGNANCY [Published IN RAMB 2015; 61(3)]}

1. What is the level of evidence of studies on birth route in case of cesarean section in a previous pregnancy? Observational longitudinal studies and lack of controlled studies (Alternative $\mathbf{A}$ ).

2. The longitudinal observational studies comparing birth route in patients with cesarean section in a previous pregnancy are characterized by being: Heterogeneous in terms of methodology to measure outcomes and presenting distinct follow-up durations (Alternative $\mathbf{A}$ ).

3. What are the characteristics associated with a favorable progress of trial of labor in patients with previous cesarean section?

Indication of the first cesarean section for non-recurring factor and vaginal delivery history, in the case of multiparous women with one previous cesarean section (Alternative $\mathbf{A})$.

4. Trial of labor in patients with previous cesarean section is:

A reasonable choice for pregnant women with one previous cesarean section performed by means of transverse uterine section (Alternative $\mathbf{A}$ ).

5. Which is the birth route indicated for patients with cesarean section in a previous pregnancy? There are still doubts about which type of delivery is better (Alternative A). 